\title{
HIGH RECOMBINATION AND MUTATION RATES IN MOUSE HEPATITIS VIRUS SUGGEST THAT CORONAVIRUSES MAY BE POTENTIALLY IMPORTANT EMERGING VIRUSES
}

\author{
Ralph S. Baric, Kaisong Fu, Wan Chen, and Boyd Yount \\ Department of Epidemiology \\ University of North Carolina \\ Chapel Hill, North Carolina 27599-7400
}

\section{INTRODUCTION}

Coronaviruses are common respiratory and gastrointestinal pathogens of mammals and birds. Not only do they cause about $15-20 \%$ of the common colds in humans, they are also occasionally associated with infections of the lower respiratory tract and central nervous system $^{1}$. The prototype, mouse hepatitis virus (MHV), contains a $32 \mathrm{~kb}$ genomic RNA which encodes two large orfs at the $5^{\prime}$ end, designated orf $1 \mathrm{a}$ and orf $1 \mathrm{~b}$. Orf $1 \mathrm{~b}$ contains highly conserved polymerase, helicase and metal binding motifs typical of viral RNA polymerases while orf 1 a contains membrane and cysteine rich domains, and serine-and poliovirus $3 \mathrm{c}$-like protease motifs ${ }^{1}$. The large size of the genome coupled with it's unique replication strategy and high recombination frequencies during mixed infection predict a considerable capacity to evolve ${ }^{1,2,3}$.

The majority of emerging RNA viruses are probably zoonotic pathogens that bridge the species barrier and spread into the human population. The probable emergence of HIV, Hantaan, and influenza viruses from zoonotic hosts suggests that this is a natural, almost predictable phenomenon, yet we know little about the molecular mechanisms mediating virus spread between species $^{3}$. To address this question in coronaviruses, we calculated MHV polymerase error rates and RNA recombination frequencies. Further MHV's capacity to bridge the species barrier was confirmed by isolating variants which grow efficiently on nonpermissive baby hamster kidney cells in vitro. 


\section{MATERIAL AND METHODS}

Temperature sensitive $(t s)$ mutants from the group $\mathrm{F} \mathrm{RNA}^{+}$mutants (NC6, NC16), group E (LA18) and group C (NC3) RNA mutants were used in this study ${ }^{2,3}$. MHVA59/JHM was adapted to baby hamster kidney cells (BHK) by serial passage in progressively increasing ratio's of BHK/DBT mixed cultures.

\section{RESULTS}

\section{Sequence Analysis of TS and Revertent Viruses}

Identification of the mutant allele in $t s$ virus in $\mathrm{MHV}$ is complicated by the large size of the viral genome including the $\sim 22 \mathrm{~kb}$ polymerase region encoding at least 5 or more genetic functions ${ }^{2}$. To simplify this problem, we focused on the $\mathrm{RNA}^{+}$mutants which mapped in the S gene of MHV (NC6, NC16) and the RNA ${ }^{-}$mutants which mapped in the $\mathrm{n}$-(NC3) or c-termini (LA18) of orf $1 \mathrm{~b}^{2,3}$. Since infectious vectors are not available, revertent viruses were isolated to assist in the identification of the mutant allele. Revertent viruses had similar titers and were of the $\mathrm{RNA}^{+}$phenotype when assayed at both permissive and restrictive temperatures (Table 1).

Overlapping primer pairs were developed to clone $1.0-1.5 \mathrm{~kb}$ stretches of viral RNA in the MHV orf $1 \mathrm{~b}$ and $\mathrm{S}$ genes using PCR ${ }^{5}$. To obviate PCR-induced mutations and circumvent high RNA polymerase error rates $^{4}$, the PCR product was reamplified by asymmetric PCR, and sequenced directly. For the $\mathrm{RNA}^{+}$mutants (NC6, NC16), the entire S glycoprotein gene was sequenced in $t s$ and revertent viruses. For the RNA- mutants (LA18, $\mathrm{NC} 3$ ), the entire orf $1 \mathrm{~b}$ region and the c-terminal 1-2 $\mathrm{kb}$ of orf $1 \mathrm{a}$ was sequenced.

NC6 contained a single nucleotide substitution at the $5^{\prime}$ end of the S glycoprotein gene which resulted in an A to G transition at position 630 (Thr to Ala; amino acid 207). $\mathrm{NC} 16$ contained a single nucleotide change involving a $\mathrm{C}$ to $\mathrm{G}$ transversion at $\mathrm{nt} 2511$ (Asp to Glu) in the S2 domain of the S glycoprotein gene. In revertents of $\mathrm{NC16}$, the mutation had reverted back to wildtype sequence (Table 2).

Among the group E RNA- mutants, LA18 contained a single nucleotide substitution at $\mathrm{nt} 7100$ in orf $1 \mathrm{~b}$. The $\mathrm{G}$ to A alteration led to a Arg to Lys change at amino acid 2286. In ten LA 18 revertants, the mutation had reverted to the wildtype sequence. The group $\mathrm{C}$ mutant NC3 contained two alterations at nt 171 and 172; located upstream from the ribosomal

Table 1. $T s$ and revertant virus titers

\begin{tabular}{lccc}
\hline & \multicolumn{3}{c}{ Titer at Temperature $\left({ }^{\circ} \mathrm{C}\right)$} \\
\cline { 2 - 4 } Ts mutant & 32 & 39.5 & $39.5 / 32$ \\
\hline LA18 & $6.1 \times 10^{7}$ & $2.4 \times 10^{3}$ & $4.0 \times 10^{-5}$ \\
LA18R1 & $2.3 \times 10^{7}$ & $9.5 \times 10^{7}$ & $4.0 \times 10^{-1}$ \\
NC3 & $2.4 \times 10^{8}$ & $5.5 \times 10^{4}$ & $2.3 \times 10^{-4}$ \\
NC3R1 & $2.0 \times 10^{8}$ & $2.0 \times 10^{8}$ & $1.0 \times 10^{0}$ \\
NC6 & $2.8 \times 10^{7}$ & $3.5 \times 10^{3}$ & $1.2 \times 10^{-4}$ \\
NC6R1 & $2.3 \times 10^{7}$ & $2.4 \times 10^{7}$ & $1.0 \times 10^{0}$ \\
NC16 & $8.5 \times 10^{7}$ & $2.3 \times 10^{4}$ & $2.7 \times 10^{-4}$ \\
\hline
\end{tabular}


Table 2. Nucleotide locations of the MHV-A59 $t s$ alleles

\begin{tabular}{|c|c|c|c|c|c|c|}
\hline \multirow[b]{2}{*}{ TS mutant } & \multirow{2}{*}{$\begin{array}{l}\text { Location in } \\
\text { genome }\end{array}$} & \multirow[b]{2}{*}{ nt site } & \multicolumn{2}{|c|}{ nt change } & \multicolumn{2}{|c|}{ AA change } \\
\hline & & & $w^{a} t^{a}$ & $\mathrm{ts}^{\mathrm{b}} / \mathbf{R e v}^{\mathrm{c}}$ & wt & $\operatorname{ts}(\operatorname{Rev})$ \\
\hline \multicolumn{7}{|l|}{ Group F } \\
\hline NC6 & $S$ gene & 620 & A & G & Tyr & Cys \\
\hline NC6R1 & & - & - & A & - & Tyr \\
\hline $\mathrm{NC16}$ & & 2502 & $\mathrm{C}$ & G & Asp & Glu \\
\hline $\mathrm{NC} 16 \mathrm{R} 1$ & & - & - & $\mathrm{C}$ & - & Asp \\
\hline \multicolumn{7}{|l|}{ Group C } \\
\hline NC3 & ORFla & 171,172 & $\mathrm{GC}$ & CG & Gly & Ala \\
\hline NC3R1 & & - & - & GC & - & Gly \\
\hline \multicolumn{7}{|l|}{ Group E } \\
\hline LA18 & ORF1b & 7100 & G & A & Arg & Lys \\
\hline LA18R1 & & - & - & G & - & Arg \\
\hline
\end{tabular}

${ }^{\text {a wildtype. }}$

$b_{\text {temperature sensitive mutant. }}$

${ }^{c}$ revertant.

frameshifting site in orf 1a. The alteration (GC to CG) resulted in a Gly to Ala at amino acid 4438. In revertant virus, reversion to wildtype sequence had occurred.

Additional nucleotide changes were also found in $t s$ and revertant viruses which were not tightly linked to the $t s$ phenotype (data not shown).

\section{Establishing Precise Recombination Rates in the MHV A59 Genome}

All mutants were crossed 3-5 X to the other mutants used in the study and standardized to the standard cross (Table 3$)^{2,3}$. To establish recombination frequencies between highly defined alleles, the nucleotide distances between individual mutants was divided by the recombination frequencies between each particular cross. Within the S gene (NC6 $\mathrm{x}$ $\mathrm{NC} 16$ ), the average recombination frequency was $1 \% \mathrm{RF} / 629 \pm 331 \mathrm{bp}$ as compared to $1 \%$ / $8979 \pm 1191 \mathrm{bp}$ in the polymerase gene (LA18 $\times$ NC3). Between the $5^{\prime}$ end of the S glycoprotein gene (NC6) and the $3^{\prime}$ end of the polymerase gene (LA18), the average recombination frequency was about $1 \% / 1054 \pm 139 \mathrm{bp}$, indicating that progressively increasing recombination frequencies were evident from the $5^{\prime}$ to $3^{\prime}$ end of the genome ${ }^{3}$.

\section{RNA Polymerase Error Rates during MHV Infection}

MHV RNA polymerase error rates were calculated from the average reversion frequencies of several $t s$ mutants and approached $1.32 \pm 0.89 \times 10^{-4}$ or about 2.4 mutations (range 1.5-7.2) per genome round of replication.

Table 3. Recombination frequencies between $t s$ mutants ${ }^{\mathrm{a}}$

\begin{tabular}{lcccc}
\hline Mutant & NC3 & LA18 & NC6 & NC16 \\
\hline NC3 & - & $0.84 \pm 0.21$ & $5.50 \pm 2.10$ & ND \\
LA18 & & - & $0.90 \pm 0.20$ & $4.6 \pm 0.60$ \\
NC6 & & & - & $2.8 \pm 0.90$ \\
NC16 & & & & - \\
\hline
\end{tabular}

amean \pm standard deviation. ND, not done. 
Table 4. Characterization of MHV BHK-adapted virus isolates

\begin{tabular}{cll}
\hline & \multicolumn{2}{c}{ Virus titers $^{\mathrm{a}}$} \\
\cline { 2 - 3 } & \multicolumn{1}{c}{ DBT } & BHK \\
\hline Virus stocks & $7.3 \times 10^{8}$ & 0 \\
MHV-A59 & $3.5 \times 10^{7}$ & $3.0 \times 10^{7}$ \\
MHV-H1 & $1.0 \times 10^{8}$ & $7.5 \times 10^{7}$ \\
MHV-H2 & & \\
Virus Growth & & $4.8 \times 10^{4}$ \\
MHV-A59 (0h) & $2.6 \times 10^{4}$ & $1.7 \times 10^{3}$ \\
MHV-A59 (24h) & $7.0 \times 10^{7}$ & $2.5 \times 10^{4}$ \\
MHV-H2 (0h) & $1.0 \times 10^{4}$ & $1.1 \times 10^{8}$ \\
MHV-H2 (24h) & $1.0 \times 10^{6}$ & \\
\hline
\end{tabular}

${ }^{\mathrm{a}}$ virus stocks titered on DBT or BHK cells.

${ }^{b}$ cultures of DBT or BHK cells were infected with virus and titer on DBT cells.

\section{MHV Evolution in Vitro}

The high rate of recombination coupled with a high RNA polymerase error rate suggested that MHV has considerable potential to evolve rapidly in vitro. Since most emerging viruses are thought to be zoonotic pathogens that bridge the species barrier ${ }^{4}$, we
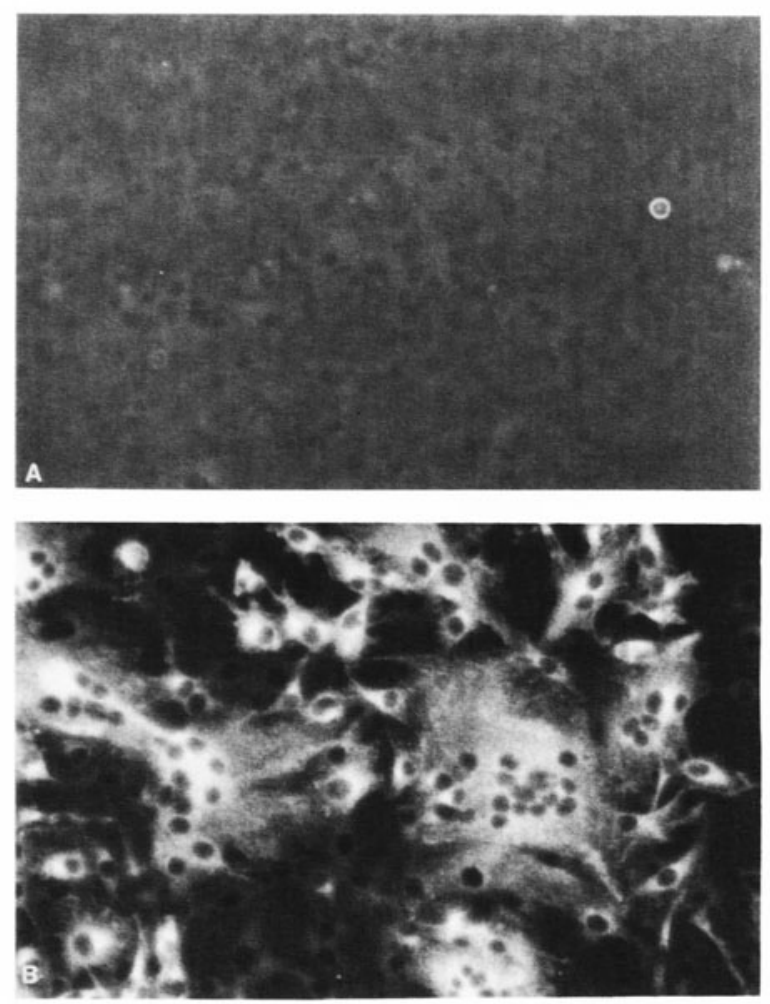

Figure 1. Expression of MHV-A59 (Panel A) and MHV-A59H2 (Panel B) viral antigen in BHK cells. 
examined the capacity of MHV to bridge the species barrier and grow in nonpermissive BHK cells. Cultures of mixed BHK/DBT cells were infected with MHV and the progeny serially passaged in progressively increasing ratios of BHK to DBT cells (1 to 10 , to 5 to 1). Following additional selection, two MHV variants (MHV-H1, MHV-H2) were isolated which replicated, expressed viral antigen, fused BHK cells, and plaque in BHK and DBT cells efficiently (Figure 1) (Table 4). Under identical conditions, MHV-A59 wild type virus did not replicate or express significant quantities of viral protein in BHK cells.

\section{DISCUSSION}

$T s$ and revertant viruses were sequenced to identify specific mutations which correlated with the $t s$ phenotype, map the location of the MHV complementation groups, determine RNA polymerase error rates and precise RNA recombination frequencies throughout the genome. While this approach suffers from the practical limits of sequencing the entire $32 \mathrm{~kb}$ genome, each mutation was uniquely present in $t s$ but not in wildtype or revertent viruses, and mapped to a domain as predicted by standard genetic recombination mapping techniques ${ }^{2}$. Although more $t s$ mutants must be sequenced, these data suggest that the group $\mathrm{F} \mathrm{RNA}^{+}$mutants map within the $\mathrm{S}$ glycoprotein gene of MHV while the group E RNAmutants, which are defective in positive strand synthesis after temperature shift, reside near the c-terminus of orf $1 \mathrm{~b}$. The group $\mathrm{C}$ mutants, which are defective in negative but not positive strand RNA synthesis, probably map at the orf $1 \mathrm{a} /$ orf $1 \mathrm{~b}$ junction.

It is well documented that viral RNA-dependent RNA polymerases do not contain proofreading activities and have high mutation rates approaching $10^{-3}$ to $10^{-5}$ per round of replication ${ }^{4}$. The resulting mixed virus populations, quasi-species, is probably an important mechanism of RNA virus evolution and spread between species. From the reversion frequencies of highly plaque purified $t s$ mutants, we determined the average MHV RNA polymerase error rate to approach $1.32 \pm 0.89 \times 10^{-4}$, or about 2.4 mutations per genome round of replication (range, 1.5-7.4). High mutation rates in a genome that is 3-4 X larger than most other positive-stranded RNA viruses suggests that coronaviruses probably exist in large quasispecies populations, providing vast reservoirs of natural virus variants.

Previous studies in our laboratory and others have suggested that RNA recombination occurs at high frequency and varies in different portions of the MHV genome ${ }^{1,2,3}$. The physical map locations of several MHV ts mutants provides accurate estimates of the homologous RNA recombination frequencies in the MHV genome which approach $1 \% / 8979 \pm 1191$ bp or about $4.9 \%$ throughout the $22 \mathrm{~kb}$ polymerase region (assuming reciprocal crosses). Between the polymerase and $\mathrm{S}$ glycoprotein genes, the recombination frequency is significantly increased, and approaches $1 \% / 1054 \pm 139 \mathrm{bp}(\sim 3.8 \%$ in the $2.0 \mathrm{~kb}$ $\mathrm{p} 30 / \mathrm{HE}$ genes). In the $4.0 \mathrm{~kb} \mathrm{~S}$ glycoprotein gene, RNA recombination rates approach $1 \% / 629 \pm 331$ bp $(\sim 12.7 \%)$. If recombination frequencies at the $3^{\prime}$ end of the genome approaches or exceeds rates measured in the $\mathrm{S}$ gene, the overall recombination rate in MHV probably exceeds $25 \%$. Currently, the most likely model to explain the high, progressively $5^{\prime}$ to $3^{\prime}$ increased recombination rate in MHV is from: (1) the large size of the genome, (2) discontinuous transcription, and (3) the presence of transcriptionally active full and subgenomic length positive- and negative-stranded RNAs which increase the amount of template for strand switching ${ }^{2,3}$.

The high rate of coronavirus RNA recombination coupled with high RNA polymerase error rates provides coronaviruses with a natural mechanism for rapid antigenic variation and evolution, especially within the highly immunogenic structural genes. It is clear that MHV can rapidly alter its species specificity and infect rats and primates; the resulting virus variants are associated with demyelinating diseases in these alternative species ${ }^{5}$. Newly 
recognized animal coronaviruses include porcine epidemic diarrhea coronaviruses which probably evolved by mutation and recombination from the human coronavirus $229 \mathrm{E}^{6}$. We have also isolated MHV variants which efficiently replicate not only in BHK cells, but to a lesser extent, have retained their natural ability to replicate in mouse cells in vitro. These data further demonstrate the capacity of coronaviruses to evolve rapidly and bridge the species barrier in vitro. Using these variants, we can map determinants which mediate species specificity, map MHV virulence markers in the hamster and/or mouse, determine if the BHK $\mathrm{CE}$ homologue or other cellular proteins acts as a receptor in alternative hosts, and determine evolution rates in structural and nonstrucural genes. The data presented in this paper demonstrates that coronaviruses can evolve rapidly and transverse the species barrier in vitro.

\section{ACKNOWLEDGMENTS}

This research was supported by grants from the American Heart Association (AHA 90-1112), the NIH (AI-23946) and was performed during the tenure of an Established Investigator Award from the American Heart Association (RSB) (AHA 89-0193).

\section{REFERENCES}

1. Lai, M.M.C. 1990. Coronavirus: organization, replication and expression of genome. Annu. Rev. Microbiol. 44, 303-333.

2. Baric, R.S. Fu, K.S., Schaad, M.C. and Stohlman, S.A. 1990. Establishing a genetic recombination map for MHV-A59 complementation groups. Virology 177: 646-656.

3. Fu, K. and Baric, R.S. 1992. Evidence for variable rates of recombination in the MHV genome. Virology 189:88-102.

4. Morse, S.S. 1994. The viruses of the future? Emerging viruses and evolution. In: Morse, S.S. (eds), The Evolutionary Biology of Viruses, Raven Press, Ltd, New York.

5. Murray, R.S., et al., 1992. Coronavirus infects and causes demyelination in the primate central nervous system. Virology 188: 274-284.

6. Brigden, A., M. Duarte, K. Tobber, H. Laude, and M. Ackermann. 1993. The nucleocapsid protein gene of the porcine epidemic diarrhoea virus confirms that this virus is a coronavirus related to HCV 229E and TGEV. J. Gen. Virol. 74:1795-1804. 